\author{
Anahi Guedes de Mello \\ Universidade Federal de Santa Catarina \\ Adriano Henrique Nuernberg \\ Universidade Federal de Santa Catarina
}

\title{
Gênero e deficiência: interseções e perspectivas
}

\begin{abstract}
Resumo: O campo de estudos feministas e de gênero tem avançado à medida que se articula com outras categorias de análise, tais como geração, classe, raça/etnia, orientação sexual, região e religião. Uma questão, contudo, permanece à revelia do esforço de interseção dessas categorias, a saber, a deficiência. Para tanto, busca-se aqui avaliar algumas das possibilidades de análise de fenômenos sociais e culturais nas quais se considera oportuno o diálogo entre os estudos feministas e de gênero com o campo de estudos sobre deficiência. Espera-se proporcionar maior visibilidade para o debate dessa questão, avaliando seu potencial analítico e político em vista da sua contribuição para as políticas públicas.

Palavras-chave: gênero; deficiência; políticas públicas.
\end{abstract}

Copyright () 2012 by Revista Estudos Feministas.

' Karla G. ADRIÃO, 2008.

${ }^{2}$ Debora DINIZ, 2003a, 2007.

\section{Introdução}

Um argumento consolidado no âmbito das Ciências Humanas e Sociais é o da necessária articulação das categorias de gênero, geração, classe, raça/etnia, orientação sexual, região e religião na análise de fenômenos sociais e culturais. Nos Estudos Feministas e de Gênero, a própria interface com os movimentos feministas demanda essa abordagem multicategorial, considerando que as questões pautadas pelo ativismo feminista, a partir da segunda geração desse movimento, transcendem aos aspectos de gênero e apontam para a relevância das demais categorias. ${ }^{1}$

Uma questão, contudo, tem sido pouco investigada nesses debates e cruzamentos intercategoriais na produção acadêmica feminista brasileira, a saber, a deficiência. ${ }^{2}$ Por ser considerada pela maior parte da comunidade acadêmica uma temática muito específica e distante, a deficiência ainda é um tema periférico nas Ciências Humanas e Sociais e particularmente no campo teórico 
${ }^{3}$ Rosemarie GARLAND-THOMSON 2002.

${ }^{4}$ Utilizamos o termo "pessoas com deficiência", pois se trata de um consenso entre as organizações oficiais e não governamentais que representam esse grupo e que vivem a condição de possuir algum impedimento de natureza física, intelectual ou sensorial, conforme corroborado por Romeu K. SASSAKI (2003). No entanto, a noção de deficiência não coincide para nós com a de impedimento ou lesão, uma vez que se trata de um processo social e cultural, conforme será explicado mais adiante.

5 Pautamo-nos no Censo Demográfico de 2010 feito pelo Instituto Brasileiro de Geografia e Estatística (IBGE). Já os dados referentes à população mundia são da World Health Organization (WHO sigla em inglês para Organização Mundial de Saúde OMS). Mais detalhes, ver em IBGE, 2011: e WHO, 2011.

${ }^{6}$ Colin BARNES, Mike OLIVER e Len BARTON, 2002; BARTON, 1998; $e$ DINIZ, 2007.

${ }^{7}$ Debora DINIZ, Flávia SQUINCA e Marcelo MEDEIROS (2007, p. 4) afirmam que "O Estado brasileiro incorporou a transversalidade de gênero e raça em grande parte das políiticas sociais, mas é ainda rara a referência à deficiência". ${ }^{8}$ Nirmala EREVELLES, 1996; Ayesha VERNON, 1999.

' Esta tem sido a tradução mais adequada e usual para o campo dos Disabilitiy Studies em nosso idioma, referendado pela maior parte das publicações que resgatam sua contribuição teórica. No Brasil, o termo equivalente para "disability" é deficiência, e não incapacidade (conforme DINIZ, MEDEIROS e SQUINCA 2007).

10 Karen P. DePAUW, 1996.

${ }^{11}$ Em Erving GOFFMAN (1988, p. 11), estigma pode ser entendido como signo, isto é, sinais ou marcas corporais "com os quais se procurava evidenciar alguma coisa de extraordinário ou mau sobre o status moral de quem os apresentava". Trata-se de um feminista. ${ }^{3}$ Ao chamarmos a atenção para esse fato, não nos referimos apenas às mais de 1 bilhão de pessoas com deficiência ${ }^{4}$ no mundo ou às cerca de 45 milhões e 600 mil em nosso país, ${ }^{5}$ mas nos pautamos na perspectiva que sustenta ser a deficiência uma condição constituinte da condição humana. ${ }^{6}$ Ademais, no Brasil, grande parte das políticas sociais para a promoção das igualdades menciona as categorias acima referidas, sem incluir a deficiência nos debates e nos cruzamentos com todas essas categorias que convivem com a experiência da opressão e da discriminação. ${ }^{7}$ Ao mesmo tempo, muitos estudos sobre o fenômeno da deficiência abordam essa temática de modo isolado, sem a necessária discussão da transversalidade da deficiência. ${ }^{8}$

Partindo dessa constatação, este trabalho pretende ressaltar a pertinência da interface entre os Estudos sobre Deficiência ${ }^{9}$ e o campo dos Estudos Feministas e de Gênero, destacando a importância desse debate para as políticas públicas. $^{10}$

Cumpre destacar que, seja como um dado empírico ou um signo, ${ }^{11}$ concebemos o fenômeno da deficiência como um processo que não se encerra no corpo, mas na produção social e cultural que define determinadas variações corporais como inferiores, incompletas ou passíveis de reparação/reabilitação quando situadas em relação à corponormatividade, isto é, aos padrões hegemônicos funcionais/corporais. Nesse sentido, a deficiência consiste no produto da relação entre um corpo com determinados impedimentos de natureza física, intelectual, mental ${ }^{12}$ ou sensorial e um ambiente incapaz de acolher as demandas arquitetônicas, informacionais, programáticas, comunicacionais e atitudinais que garantem condições igualitárias de inserção e participação social. ${ }^{13}$ Essa definição contempla a perspectiva social da deficiência e está de acordo com a letra "e" do preâmbulo da Convenção sobre os Direitos das Pessoas com Deficiência, ${ }^{14}$ quando afirma que "a deficiência resulta da interação entre pessoas com deficiência e as barreiras devidas às atitudes e ao ambiente que impedem a plena e efetiva participação dessas pessoas na sociedade". ${ }^{15}$ Desde esse ponto de vista sociocultural, deficiência englobaria os diferentes sentidos ou modos de defini-la, percebê-la, vivenciá-la, tratá-la etc., o que nos remonta às categorias nativas em torno da experiência da deficiência.

Diante do exposto, pretendemos aqui abordar algumas questões relativas à constituição social da experiência da deficiência, a partir de uma perspectiva feminista do campo dos Estudos sobre Deficiência. A abordagem tem como foco as questões de construção do 
trabalho pioneiro de Goffman, que estudou os mecanismos desencadeadores de estigmas, mostrando, sobretudo, como se processa a manipulação da identidade de pessoas com deficiência.

12 É importante diferencia deficiência mental de deficiência intelectual. Atualmente, a deficiência mental se refere às pessoas com as "sequelas de transtorno mental", de acordo com a Lei n. 10.216/2001. De fato, segundo a Convenção sobre os Direitos das Pessoas com Deficiência (BRASIL, 2008b), as pessoas com sofrimento mental também são consideradas pessoas com deficiência, sob a tipificação de "deficiência mental", para diferenciá-la de deficiência intelectual, esta última alusiva a uma parte específica do funcionamento do intelecto, e não de toda a mente.

${ }^{13}$ Debora DINIZ e Lívia BARBOSA 2010.

${ }^{14}$ Esta convenção, aprovada em 13 de dezembro de 2006 durante a Assembleia Geral da Organização das Nações Unidas (ONU), em Nova lorque, EUA, e ratificada pelo Brasil juntamente com Protocolo Facultativo à Convencão em $1^{\circ}$ de agosto de 2008 foi incorporada no ordenamento jurídico brasileiro sob o status de equivalência constitucional através do Decreto Legislativo $n$. 186/2008 (BRASIL, 2008a).

15 BRASIL, 2008b, p. 21

${ }^{16} \mathrm{~A}$ deficiência como uma categoria analítica se encontra praticamente ausente em todo o escopo das Ciências Sociais brasileiras, conforme corroborado por Diniz (2007).

17 Agustina PALACIOS, 2008; e Eduardo M. VASCONCELOS 2003.

18 BARNES, 1999; Brendan J. GLEESON, 1997; e OLIVER, 1983 e 1990.

19 Adrienne ASCH, 2004 GARLAND-THOMSON, 2002; Eva F KITTAY, 1999; Margaret LLOYD, 1992: Jenny MORRIS, 1991, 1992 e 1996; e Susan WENDELL, 1996

20 Em Lennard DAVIS (1997) corpo, do gênero e da dependência para compreender como essas categorias se articulam com a experiência da deficiência, de forma que se possa demonstrar a pertinência de novos estudos envolvendo o recorte da deficiência ${ }^{16}$ nas Ciências Humanas e Sociais.

\section{Os estudos sobre deficiência}

Ao contrário dos Estudos Feministas e de Gênero, os Estudos sobre Deficiência são pouco conhecidos no país. Em realidade, o tema deficiência ganha no Brasil um enfoque predominantemente biomédico, sendo pouco contemplado nas Ciências Humanas e Sociais. Desse modo, julgamos ser necessário delinear algumas características desse campo, visando contextualizar o enfoque aqui proposto sobre a relação gênero e deficiência.

Os Estudos sobre Deficiência emergiram nos anos 1970 e 1980 em decorrência das lutas políticas, nos anos 1960 e 1970, das pessoas com deficiência nos Estados Unidos (Independent Living Movement), na Inglaterra (Union of the Physically Impaired against Segregation) e nos países nórdicos (Self-advocacy Movement, na Suécia), ${ }^{17}$ primeiro com uma perspectiva histórico-materialista ${ }^{18} \mathrm{e}$, após, desde o início dos anos 1990, com a contribuição da epistemologia feminista. ${ }^{19}$ Esse campo vem despontando como um sólido contexto acadêmico interdisciplinar que pretende refletir, em suas mais variadas vertentes, ${ }^{20}$ sobre o fenômeno da deficiência a partir do uso de metodologias e ferramentas analíticas próprias das Ciências Sociais. Vários são os programas de graduação e/ou pósgraduação que já esboçam essa proposta em muitos países, ${ }^{21}$ mas ainda não têm significativa presença nas Ciências Sociais brasileiras, permanecendo restrita a outras áreas do conhecimento, como a Psicologia, a Educação e a Medicina.

No Brasil, a interface do feminismo com os Estudos sobre Deficiência tem sido feita de modo intenso e com amplo destaque nacional pela Anis - Instituto de Bioética, Direitos Humanos e Gênero, ${ }^{22}$ sendo a primeira organização não governamental, sem fins lucrativos, voltada para a pesquisa, o assessoramento e a capacitação em Bioética na América Latina. Sediada em Brasília e com uma equipe permanente de profissionais de diferentes áreas, a Anis também desenvolve ações de capacitação e produção de conhecimento em torno das questões de bioética e deficiência, em parceria com o grupo de pesquisa Ética, Saúde e Desigualdade da Universidade de Brasília (UnB). Uma boa parte das referências nacionais aqui utilizadas é de autoria desse grupo, no qual participam pessoas de 
propõe-se uma revisão dos Estudos sobre Deficiência a fim de ampliar o conceito de deficiência nas perspectivas marxistas, feministas, étnicas, culturais, pós-coloniais e pósmodernas.

${ }^{21}$ O seguinte link mostra uma lista de outros links externos indicando sites de jornais ou periódicos, sociedades científicas e universidades com programas universitários em Estudos sobre Deficiência: $<$ http://en.wikipedia.org/wiki/ Disability_studies\#External_links> A principal referência nesse campo é o Centro de Estudos sobre Deficiência da Universidade de Leeds, na Inglaterra: < http:// www.leeds.ac.uk/disability-studies/> 22 Confira o site <http:// www.anis.org.br $>$, em que se detalham os objetivos e os trabalhos dessa importante organização.

${ }^{23}$ Patricia BROGNA, 2005; e DINIZ, 2007.

${ }^{24}$ Bruno Daniel G. S. MARTINS 2005, p. 11. Ver também a crítica de OLIVER (1986) à "teoria da tragédia pessoal".

${ }^{25}$ DINIZ, 2007, p. 23.

${ }^{26}$ DINIZ, 2003a e 2007. grande renome nacional, como a antropóloga Debora Diniz, pioneira na difusão do modelo social da deficiência no Brasil e da interface dessa questão com as teorias feministas por meio do livro O que é deficiência?, da editora Brasiliense, entre outras publicações referenciadas ao longo deste texto.

De modo geral, o modelo social da deficiência, em oposição ao paradigma biomédico, não se foca nas limitações funcionais oriundas de deficiência nem propõe a ideia tão comumente aceita da necessidade de reparação/reabilitação do corpo deficiente, ${ }^{23}$ mas sim a concebe como o resultado das interações pessoais, ambientais e sociais da pessoa com seu entorno. Nesse sentido, as experiências de opressão vivenciadas pelas pessoas com deficiência não estão na lesão corporal, mas na estrutura social incapaz de responder à diversidade, à variação corporal humana.

Mesmo diante dessa tensão entre natureza e cultura, o modelo social da deficiência promoveu a primeira guinada ao elevar as pessoas com deficiência ao status de sujeitos de direitos humanos e não reduzindo esse grupo social como mero objeto de intervenção clínica, de reabilitação ou de assistencialismo. O modelo social da deficiência é o pilar dos Estudos sobre Deficiência. Sua teoria configura-se, portanto, na crítica radical ao modelo médico, uma vez que esse está intimamente enraizado na "teoria da tragédia pessoal como a narrativa cultural dominante [...]". ${ }^{24}$ Entretanto, é importante ressaltar que o que se questiona no modelo social é a ideia de que a deficiência é somente um fato orgânico. Ou melhor, "em um ponto os modelos social e médico coincidiam: ambos concordavam que a lesão era um tema da alçada dos cuidados médicos". ${ }^{25}$

Os significados construídos em torno de gênero e deficiência devem ser compreendidos como a relação entre o corpo com impedimento e o poder, sendo frutos de disputas e/ou consensos entre os diversos saberes, e não algo dado, estático e natural. Na esteira desse argumento é que se resgatam os Estudos Feministas e de Gênero. Conforme aponta Diniz, ${ }^{26}$ a primeira geração dos Estudos sobre Deficiência era composta majoritariamente de homens com lesão medular, apontados pelas teóricas feministas como membros da elite dos deficientes e, portanto, reprodutores dos discursos dominantes de gênero e classe na sociedade. Com base nessa perspectiva feminista, nos anos 1990 e 2000 o modelo social da deficiência passa por uma nova revisão, constituindo a segunda geração de teóricos desse campo:

Foram as teóricas feministas que, pela primeira vez, mencionaram a importância do cuidado, falaram sobre a experiência do corpo doente, exigiram uma 
${ }^{27}$ DINIZ, 2007, p. 3-4, grifos nossos. ${ }^{28}$ Carolyn L. VASH, 1988; e WENDELL, 1996.

${ }^{29}$ VASCONCELOS, 2003.

${ }^{30}$ Por exemplo, ver em Jessica SCHEER, 1995.

31 DINIZ, 2003a e 2007; e Francisco ORTEGA, 2009.

${ }^{32}$ Mark SHERRY, 2004.

${ }^{33}$ Mairian CORKER e Tom SHAKESPEARE, 2002.

${ }^{34}$ Jennifer M. MAYS, 2006; e Lúcia C. A. WILLIAMS, 2003. A Convenção sobre os Direitos das Pessoas com Deficiência faz menção ao gênero sete vezes e ainda inclui em seu texto um artigo específico (Artigo 6) sobre as mulheres com deficiência, em razão de sua dupla condição de vulnerabilidade por gênero e deficiência. ${ }^{35} \mathrm{ASCH}, 2004$; Beth A. FERRI e Noel GREGG, 1998; Michelle FINE e Adrienne ASCH, 1988; Anne FINGER, 1992; dentre outros.

${ }^{36}$ Ver também em GARLANDTHOMSON, 2002

${ }^{37}$ Thomas J. GERSCHICK, 2000; e VERNON, 1999

38 FERRI e GREGG, 1998, p. 429

39 "O status de mulheres com deficiência ressalta o seu duplo silêncio e opressão. O duplo silêncio de mulheres com deficiência se evidencia através da falta de uma perspectiva da deficiência na teoria e prática feminista, e na carência dos movimentos de direitos da deficiência em analisar como o gênero apresenta impacto sobre a deficiência" (FERRI e GREGG, 1998, p. 429 , tradução livre nossa). Nos Disability Studies esse duplo enfoque está embasado na Teoria da Dupla Discriminação. Para mais detalhes, ver também Asunción M. MAYA, 2004. discussão sobre a dor e trouxeram os gravemente deficientes para o centro das discussões - aqueles que jamais serão independentes, produtivos ou capacitados à vida social, não importando quais ajustes arquitetônicos ou de transporte sejam feitos. Foram as feministas que introduziram a questão das crianças deficientes, das restrições intelectuais e, o mais revolucionário e estrategicamente esquecido pelos teóricos do modelo social, o papel das cuidadoras dos deficientes. Foi o feminismo quem levantou a bandeira da subjetividade na experiência do corpo lesado, o significado da transcendência do corpo para a experiência da dor, forçando uma discussão não apenas sobre a deficiência, mas sobre o que significa viver em um corpo doente ou lesado. ${ }^{27}$

É essa abordagem feminista que interessa aos propósitos deste trabalho. A manifestação de uma deficiência evidencia essa possibilidade de transformação social, de transcendência do sujeito aos seus limites corporais. ${ }^{28}$ Trata-se, conforme prenuncia o tífulo de um livro de Vasconcelos, ${ }^{29}$ do "poder que brota da dor e da opressão".

Nesse sentido, o impacto causado pelos Estudos Culturais $^{30}$ e pelas teorias feministas, ${ }^{31}$ queer ${ }^{32}$ e pósmodernas ${ }^{33}$ também se refletiu nos Estudos sobre Deficiência, especialmente em relação às discussões teóricas sobre os modelos da deficiência e à deficiência como uma condição de vulnerabilidade para a violência de gênero. ${ }^{34}$ Trabalhos de feministas ${ }^{35}$ merecem destaque tanto por problematizarem, implícita ou explicitamente, a relação entre deficiência e gênero quanto por apontarem para uma total falta de preocupação dos movimentos feministas com a questão da deficiência; ${ }^{36}$ e dos movimentos de pessoas com deficiência em relação à importância de outras categorias identitárias, em especial a de gênero, igualmente significativas para a formação da identidade das pessoas com deficiência.$^{37}$ Tomando o exemplo do trabalho de Ferri e Gregg, ${ }^{38}$ as mulheres com deficiência têm sido historicamente negligenciadas por esses dois movimentos:

The status of women with disabilities underscores their dual silence and oppression. The dual silence of women with disabilities is evidenced by a lack of a disability perspective in feminist theory and practice, and a failure of the disability rights movement to analyze how gender impacts on disability. ${ }^{39}$

Para essas teóricas feministas, as mulheres com deficiência estão em dupla desvantagem devido a uma complexa combinação de discriminação baseada em gênero e deficiência. Consequentemente, enfrentam uma 
${ }^{40}$ ADRIÃO, 2008; e Miriam P. GROSSI, 1998.

${ }^{41}$ Três trabalhos fornecem um panorama mais detalhado acerca do nascimento dos Estudos sobre Deficiência, a saber: BARTON E OLIVER, 1997; DINIZ, 2007; e PALACIOS, 2008. Desses três, consideramos o de Palacios o mais completo.

${ }^{42}$ GARLAND-THOMSON, 2002.

${ }^{43}$ KITTAY, 1999. situação peculiar de dupla vulnerabilidade, que se torna ainda mais complexa a partir da incorporação das categorias de raça/etnia, classe, orientação sexual, geração, região e religião.

As lutas dos movimentos sociais de pessoas com deficiência guardam um ponto em comum com os movimentos feministas e de lésbicas, gays, bissexuais, travestis, transexuais, transgêneros e intersexuais (LGBTTI), que é o de questionar o construto do corpo como um dado natural que antecede a construção dos sujeitos. Portanto, da mesma forma como ocorreu com os Estudos Feministas e de Gênero em relação às histórias de lutas do feminismo, ${ }^{40}$ não se pode tratar dos Estudos sobre Deficiência sem recorrermos à história dos movimentos da deficiência. ${ }^{41}$ Foram os ativistas com deficiência os principais responsáveis pela construção e pela consolidação dos Estudos sobre Deficiência como um projeto político-acadêmico.

\section{Interfaces conceituais entre os dois campos}

Do ponto de vista conceitual é possível identificar alguns eixos importantes de articulação entre os campos dos Estudos Feministas e de Gênero e os Estudos sobre Deficiência: 1) o pressuposto da desnaturalização do corpo; 2) a dimensão identitária do corpo; e 3) a ética feminista da deficiência e do cuidado.

A respeito do primeiro pressuposto teórico, pode-se dizer que os Estudos Feministas e de Gênero têm se tornado uma ferramenta conceitual importante para a radicalização do argumento da construção social da deficiência, compreendida a partir desse campo como uma narrativa produzida socialmente sobre determinadas variações corporais. Rosemarie Garland-Thomson é uma das mais expressivas autoras feministas responsáveis pela defesa dessa ideia. ${ }^{42} \mathrm{Em}$ relação ao segundo pressuposto, consideramos que a deficiência também pode ser pensada na perspectiva da genealogia do sujeito modernocontemporâneo, da centralidade do corpo deficiente como idioma simbólico e político, da identidade e seu impacto na subjetividade da pessoa, concepção essa bastante cara à teoria feminista. Sobre o terceiro e último pressuposto, a matriz epistemológica feminista dos Estudos sobre Deficiência se destaca, sobretudo, pela frase provocativa de Eva Kittay, uma filósofa que se colocou na posição de cuidadora de uma filha com paralisia cerebral: "Todos somos filhos de uma mãe". Ou seja, essa autora trouxe à tona o tema do cuidado para além de uma questão de gênero, também um princípio ético e moral da própria condição humana, desde o nosso nascimento até a morte. ${ }^{43}$ 
${ }^{44}$ TOM SHAKESPEARE, 1999.

${ }^{45}$ BRASIL, 2010.

${ }^{46}$ FERRI e GREGG, 1998; Gisela HELMIUS, 2001: MORRIS, 1991; Diane RANDOLPH e Elena Marie ANDRESEN, 2004; e Ellen SAMUELS, 2002

${ }^{47}$ VERNON, 1999.

${ }^{48}$ Amir B. BEIGI e Karen K. Y. CHENG, 2010.
Cabe agora refletir brevemente sobre exemplos do potencial analítico da articulação dessas duas categorias como contribuição ao pressuposto da transversalidade de ambas e seu valor para a análise de fenômenos socioculturais.

\section{O potencial analítico da interseção gênero e deficiência}

É impossível esgotar aqui todas as possibilidades analíticas da interseção gênero e deficiência ou ainda mapear integralmente a riqueza da interface dos Estudos Feministas e de Gênero com os Estudos sobre Deficiência. No entanto, identificamos na sequência algumas questões em que essa interseção se faz profícua, quando possível, apontando a relevância desse diálogo para o redimensionamento das políticas públicas.

\section{Masculinidades, feminilidades e deficiência}

Como temos demonstrado, a deficiência pode ser compreendida como uma experiência marcada por processos de gênero. Shakespeare, ${ }^{44}$ revisando a literatura a respeito desse tema, comenta que feminilidade e deficiência reforçam-se mutuamente, enquanto que deficiência e masculinidade contradizem-se entre si, justamente pelos estereótipos associados a essas formas hegemônicas de identidades, a partir do binômio atividade/ passividade. Aliás, a própria forma hegemônica de masculinidade é geradora de deficiência, uma vez que remete os homens a atitudes de risco e violências que resultam muitas vezes na aquisição de alguma deficiência, dado importante para as emergentes propostas de saúde do homem nas políticas públicas na área de saúde. ${ }^{45}$

Por outro lado, no caso das mulheres, é recorrente na literatura feminista o argumento que evidencia a "dupla desvantagem" com que vivem as mulheres com deficiência em relação a participação social, direitos sexuais e reprodutivos, educação, trabalho e renda. ${ }^{46} \mathrm{Ao}$ se constituírem mutuamente e se retroalimentarem, os efeitos do duplo estigma potencializam a exclusão das mulheres com deficiência, processo que se complexifica ainda mais quando cruzado com outras categorias como raça/etnia e classe. ${ }^{47}$ De todo modo, o que se quer ressaltar aqui é que, se tendemos hoje a falar de masculinidades e feminilidades, é preciso ressaltar a deficiência como componente do espectro de possibilidades dessas posições de gênero plurais.

Com base em Beigi e Cheng ${ }^{48}$ podemos ainda problematizar o sistema binário de sexo/gênero que polariza homens e mulheres e apontar para o espectro de 
${ }^{49}$ Eva F. KITTAY, Bruce JENNINGS e Angela A. WASUNNA, 2005.

50 DINIZ, 2007; Raque GUIMARÃES, 2010; e SQUINCA, 2008.

${ }^{51}$ MORRIS, 2001.

${ }^{52}$ KITTAY, 1999; e KITTAY, JENNING e WASUNNA, 2005.

${ }^{53}$ DINIZ, 2007.

${ }^{54}$ Termo cunhado por Eva Kittay (1999).

${ }^{55}$ MORRIS, 2001.

${ }^{56}$ GUIMARÃES, 2010, p. 208. possibilidades que a esfera da sexualidade admite, incluindo o segmento LGBTTI nesse debate. Isso nos leva a problematizar a própria ideia de dupla vulnerabilidade das mulheres com deficiência a partir da consideração da diversidade que comporta esse grupo, não apenas em relação à classe e à raça/etnia, mas também em relação à orientação sexual.

\section{Deficlêncla, culdado e feminlsmo}

Como já afirmado, foi mérito das feministas a crítica à perspectiva individualista da primeira geração dos Estudos sobre Deficiência, que negava a dimensão do cuidado, negligenciando a condição de pessoas com graves lesões que dependem do auxílio de outros nas atividades da vida diária, mobilidade, participação social. À medida que promove a visibilidade à dimensão do cuidado como uma questão de justiça, ${ }^{49}$ a perspectiva feminista da deficiência permite politizar esse contexto da vida privada, bem como resgata a condição da mulher cuidadora, muitas vezes esquecida no bojo das políticas públicas para mulheres e pessoas com deficiência. ${ }^{50}$ Ademais, como nos alerta Jenny Morris, ${ }^{51}$ é preciso evitar reproduzir as análises que dividem, ainda que pautadas por argumentos feministas, a relação de cuidado entre as "mulheres cuidadoras" e seus "dependentes", sendo esses excluídos de sua condição de gênero. A transversalidade de gênero e deficiência nessa questão exige tais cuidados analíticos e conceituais.

Nesse contexto, também merece destaque a produção de Eva Kittay ${ }^{52}$ como uma das pioneiras da crítica à noção de independência proposta pela primeira geração de teóricos dos Estudos sobre Deficiência, trazendo em seu lugar a noção de interdependência como um valor humano que se aplicava a pessoas com e sem deficiência. ${ }^{53}$ Como filósofa e cuidadora de uma filha com deficiência, soube defender que a dependência do outro é parte da condição humana e, no caso de muitas pessoas com deficiência, a garantia do cuidado é um direito fundamental para a manutenção da vida e a conquista da dignidade humana. Isso nos obriga, assim, a pensar o cuidado como uma responsabilidade do Estado e da sociedade, desnaturalizando essa atividade como naturalmente feminina e propondo uma ética do cuidado ${ }^{54}$ que se paute nos direitos humanos e reconheça a deficiência (incluindo aí a dependência) como condição inerente à diversidade humana. ${ }^{55}$ Nesse sentido, concordamos com Raquel Guimarães, ${ }^{56}$ quando afirma que,

Em sociedades que não reconhecem a dependência, frequentemente a pessoa que cuida é explorada e 
${ }^{57}$ KITTAY, JENNINGS e WASUNA, 2005.

${ }^{58}$ Anahi G. MELLO, 2010.

${ }^{59}$ BARNES, OLIVER E BARTON, 2002 DINIZ, 2007; e Bill HUGHES, 2002.

${ }^{60}$ Charles GARDOU, 2006.

${ }^{61}$ Sônia W. MALUF, 2001, p. 96

${ }^{62}$ GARLAND-THOMSON, 2009 invisibilizada. $O$ trabalho do cuidado ocupa uma posição inferior em uma hierarquia de gênero que organiza a vida em sociedade, por isso não há políticas públicas voltadas ao cuidado, tampouco proteção social para quem o exerce. A questão da deficiência compreendida sem a abordagem transversal de gênero produz impactos significativos na vida de pessoas que exercem o ofício do cuidado.

Verificamos, portanto, nesse argumento, um avanço importante que a interseção das categorias de gênero e deficiência pode proporcionar à reflexão tanto sobre as políticas públicas que combatem a desigualdade de gênero quanto sobre aquelas que visam à inclusão das pessoas com deficiência. Há, pois, uma proposição ética que se desdobra dessas reflexões: uma ética feminista do cuidado que transcende à questão da deficiência, que nos alerta a respeito de nossa condição de interdependência e que reconhece o valor do cuidado como uma necessidade humana, implicando uma mudança política fundamental em torno de fronteiras sociais e ideológicas para que sejam compatíveis com a noção de justiça e de direitos humanos. ${ }^{57}$

\section{Corporalidade e deficiência}

A questão da deficiência se constitui como um problema de vastas possibilidades no estudo da corporalidade como um processo sociocultural, ${ }^{58}$ ainda mais após a proposição do modelo social da deficiência da segunda geração desse campo, influenciada pelas teóricas feministas. ${ }^{59}$ No tocante ao diálogo com os Estudos Feministas e de Gênero, os Estudos sobre Deficiência podem contribuir para o avanço do pressuposto da construção social do corpo. Conforme sustenta Gardou, ${ }^{60}$ a experiência do corpo deficiente já vinha revelando dimensões novas para a abordagem antropológica do corpo e da corporalidade. A corporificação da experiência da deficiência, ao subverter o estigma do corpo com lesão, releva a condição de pessoa, ou seja, a deficiência "é também uma forma de se constituir como um determinado tipo de sujeito - nesse caso é o corpo, ou mais especificamente uma determinada corporalidade, que constrói uma determinada pessoa". ${ }^{61}$

A perspectiva feminista tem qualificado o debate sobre o corpo no contexto dos Estudos sobre Deficiência, especialmente no tocante à radicalização do pressuposto da desnaturalização do corpo. Conforme GarlandThomson, ${ }^{62}$ as teorias feministas levam esse debate para além da crítica ao corpo perfeito e às barreiras de acesso, à medida que contemplam uma análise profunda sobre o estatuto social e cultural do corpo, sobre a política da 
63 Judith BUTLER, 1993 e 2003.

${ }^{64}$ Agradecemos à Prof.a Dr.a Miriam Pillar Grossi pelo incentivo à produção deste texto e também à até então estagiária docente Rosa Blanca pela revisão dos conceitos de Butler em trabalho originalmente apresentado pela primeira autora na disciplina Sexualidades do Programa de PósGraduação em Antropologia Social da Universidade Federal de Santa Catarina, ministrada pela referida professora durante o primeiro semestre de 2010 .

${ }^{65}$ Jackie L. SCULLY, 2010.

${ }^{66}$ HUGHES, 2009.

${ }^{67}$ Mary DOUGLAS, 1976, p. 11-12

${ }^{68}$ Lígia A. AMARAL, 1995; e Otto M. SILVA, 1986.

${ }^{69}$ SAMUELS, 2002. aparência, a medicalização do corpo e da subjetividade e a construção social da identidade no contexto do corpo considerado deficiente.

Nesse contexto, tem sido cada vez mais citada a obra de Butler, ${ }^{63}$ teórica feminista cujos conceitos sobre corporalidade $^{64}$ têm sido incorporados na reflexão sobre gênero e deficiência. Embora essa autora não se refira diretamente à deficiência em suas reflexões, é cada vez mais comum entre estudiosas/os feministas da deficiência o resgate de conceitos como "performatividade" e "corpos abjetos" para se referir à condição da pessoa com deficiência. Por exemplo, Scully ${ }^{65}$ afirma que a deficiência pode ser performatizada para caber e confirmar estereótipos que constituem determinada deficiência, a fim de que as pessoas com deficiência possam ser socialmente legitimadas como deficientes e receber as ajudas necessárias. Ainda, consideramos que a deficiência remonta à performatividade queer, posto que o corpo deficiente também se enquadra dentro de um sistema de classificação e produção de sujeitos, em que o padrão de normalidade é inventado no marco das relações de assimetria e de desigualdade. Nesse sentido, os corpos deficientes também são considerados corpos queer, fazem parte do rol das corporeidades abjetas ${ }^{66}$ que ferem a ordem social e demarcam a linha proibida entre o sagrado e o profano. A possessão do demônio relaciona à deficiência o resultado do pecado e da "condição impura", a própria ideia da "sujeira [que] ofende a ordem". ${ }^{67} \mathrm{~A}$ abjeção ou repulsa que o corpo deficiente provoca nos "normais" afeta a relação com o outro e com o próprio corpo naquele que se sente diferente, adquirindo um protagonismo superlativo que se soma à exigência de encaixar o outro dentro de padrões hegemônicos antropométricos, fisiométricos e psicométricos, sendo ele exterminado ou segregado, apartado do convívio com os "perfeitos, belos e saudáveis". Assim, devido à promoção da beleza harmônica que herdamos dos gregos até nossos dias, a manifestação da deficiência lesiona o conceito e a idealização de corporeidade grega que eugenicamente temos tão enraizados dentro de nós. ${ }^{68} \mathrm{Sem}$ embargo, a despeito dos limites e dos cuidados dessa apropriação dos conceitos de Butler para os Estudos sobre Deficiência, ${ }^{69}$ anuncia-se um contexto bastante profícuo de articulação teórica, especialmente quanto à reflexão sobre a produção discursiva dos corpos deficientes e, concomitantemente, quanto ao reconhecimento da deficiência nos Estudos Feministas e de Gênero.

644 Estudos Feministas, Florianópolis, 20(3): 635-655, setembro-dezembro/2012 


\section{Deficiência, sexualidade e direitos reprodutivos}

Tema central no âmbito dos Estudos Feministas e de Gênero, a questão da sexualidade numa abordagem feminista adquire novos contornos em sua interseção com os Estudos sobre Deficiência. Ganha ainda maior relevo a crítica da biologização do corpo e da patologização da

${ }^{70}$ SHAKESPEARE, 1998.

71 SHAKESPEARE, 1999.
${ }^{72}$ FINGER (1992, p. 9) fez o mesmo questionamento: "Muitas vezes a sexualidade é a causa de nossa opressão mais grave; também muitas vezes é a causa de nossa mais profunda dor. Resulta-nos mais fácil falar - e formular estratégias para a mudança sobre a discriminação no trabalho, na educação, na moradia, que falar sobre nossa exclusão da sexualidade e da reprodução". sexualidade engendrada pela perspectiva medicalizante. ${ }^{70}$ A negação da sexualidade e do prazer da pessoa com deficiência é analisada com base na contribuição dos Estudos Feministas e de Gênero, em função da riqueza conceitual que tais estudos puderam conquistar nas últimas décadas. ${ }^{71}$ Especialmente em relação aos artigos 23 e 24 da Convenção sobre os Direitos das Pessoas com Deficiência, respectivamente sobre o "respeito pelo lar e pela família", que trata de aspectos relacionados aos direitos de contraírem matrimônio e constituírem família (inclusive o direito "à guarda, custódia, curatela e adoção de crianças [...]"), e sobre a "saúde" (inclusive no âmbito da saúde sexual e reprodutiva), é interessante observar que os movimentos sociais de pessoas com deficiência e de lésbicas, gays, bissexuais, travestis, transexuais, transgêneros e intersexuais (LGBTTTI) guardam uma grande semelhança entre si no quesito interdições da sexualidade, uma vez que historicamente lhes são negados o direito à sexualidade e o de constituírem família. Essas questões fizeram com que os governos reconhecessem a importância de se incluir nessa convenção o tema das meninas e das mulheres com deficiência, das perspectivas de gênero e da igualdade entre as mulheres e os homens com deficiência, assim como entre os homens e as mulheres sem deficiência.

Já no contexto das políticas públicas cabe um comentário especial em relação a essa temática. Consideramos que o estatuto de sujeito de direitos das pessoas com deficiência avançará somente quando o tema da sexualidade for contemplado nessas políiticas, ${ }^{72}$ uma vez que sua condição de pessoa comum será assim reconhecida. Os temas que já estão bem discutidos nos movimentos e nas políticas sociais da deficiência (Educação, Trabalho, Acessibilidade etc.) ainda pertencem à esfera pública. São os temas da esfera privada que irão garantir a maior conquista desse estatuto de pessoa, por seu efeito político singularizante. Nesse sentido, as pessoas com deficiência têm muito a aprender com os movimentos feministas e LGBTTI em suas atuais demandas em matéria de direitos sexuais e reprodutivos, que se concentram em questões do campo privado.

No que concerne à autonomia reprodutiva da mulher, podemos identificar um ponto de tensão entre os Estudos 
${ }_{73} \mathrm{ASCH}, 2003 ;$ Alessandra BARROS, 2003; e DINIZ, 2003b. ${ }^{74} \mathrm{ASCH}, 2003$.

${ }^{75} \mathrm{ASCH}, 2003$, p. 49.

${ }^{76}$ Vera PAIVA, Camila PERES e Cely BLESSA, 2002. sobre Deficiência e os Estudos Feministas e de Gênero: o aborto seletivo de fetos com má-formação e/ou alterações cromossômicas. ${ }^{73}$ Segundo Adrienne Asch, ${ }^{74}$ uma das mais expressivas teóricas da bioética feminista dos Estudos sobre Deficiência, o aborto seletivo representa uma afronta à dignidade e à integridade moral das pessoas com deficiência, devendo ser revistos os critérios biomédicos dos diagnósticos pré-natais nesse caso. Para essa autora, as modernas técnicas de diagnóstico de anomalias fetais devem levar em consideração os pressupostos do modelo social da deficiência, através de uma "intensificação das políticas de bem-estar para as pessoas com deficiência"75 que deveriam pautar sobre as escolhas do casal.

Outra questão importante com respeito à sexualidade na interface de gênero e deficiência é a constatação da maior vulnerabilidade das pessoas com deficiência ao HIV/ aids, sobretudo pela pouca consideração das políticas públicas dessa área às especificidades desse grupo social. Essa maior vulnerabilidade se assenta na presença de diversas barreiras informacionais, comunicacionais, programáticas e atitudinais que constituem suas práticas sexuais, acarretando no que Paiva e colaboradoras ${ }^{76}$ designam como "sinergia das pragas", em referência ao amálgama de fatores que constituem o sistema social e que se entrelaçam na produção das condições de vulnerabilidade ao HIV/aids entre grupos marginalizados.

\section{Violência de gênero e deficiência}

Muitas pesquisadoras que se dedicam ao estudo das violências contra as mulheres não têm dado a devida atenção quanto ao fato de que muitas mulheres que adquirem uma lesão ou impedimento por terem sido submetidas a violências de gênero passam a ser reconhecidas como pessoas com deficiência. Acreditamos que isso ocorre porque sua condição é nesse caso interpretada dentro do contexto geral das lesões corporais decorrentes de situações de violências, mas não se especifica sua natureza, o que dificulta a sua interpretação como categoria de análise. Um caso notório é o de Maria da Penha Maia Fernandes, que se tornou paraplégica em decorrência de um tiro disparado por seu até então marido. Assim, foi em homenagem a uma mulher com deficiência que a Lei n. 11.340/2006 se tornou mais conhecida como Lei Maria da Penha, a primeira lei federal dirigida à prevenção, ao enfrentamento e ao combate a todas as formas de violência contra as mulheres.

Por outro lado, cabe ressaltar o fato da maior vulnerabilidade das mulheres com impedimentos corporais 
${ }^{77}$ Dena HASSOUEH-PHILLIPS e Mary A. CURRY, 2002.

${ }^{78}$ Rosemary CALDERBANK, 2000 ao abuso sexual. Embora existam similaridades nas formas de violências sofridas por mulheres com e sem deficiência, ${ }^{77}$ há também especificidades que merecem ser avaliadas. $O$ isolamento social, a dependência de educadoras/es, cuidadoras/es e prestadoras/es de serviços, o tipo de deficiência e o grau de funcionalidade associada à deficiência, a impossibilidade de defesa física de algumas pessoas com deficiência e diversos outros impedimentos à percepção e à reação diante do abuso levam a situações de maior risco desse grupo social. Ademais, conforme afirma Calderbank, ${ }^{78}$ esse fato está diretamente vinculado às atitudes socialmente sustentadas na relação com as pessoas com deficiência, que levam ao descrédito social e à submissão dessas ao risco de violências, em que operam tanto a questão do significado social da deficiência quanto a questão de gênero. Isso evidencia o quanto os estudos sobre a violência de gênero têm a avançar ao contemplar tais problemas na pauta de suas investigações e teorizações, favorecendo o incremento das políticas públicas no enfrentamento dessas formas específicas de vulnerabilidade social.

\section{Considerações finais}

As Ciências Humanas e Sociais em geral, e em particular os Estudos Feministas e de Gênero, têm muito a ganhar ao se dedicar ao tema da deficiência, uma vez que os dispositivos e as significações sociais têm grande potencial de fazer esse campo científico avançar em seus temas mais fundamentais. Ou seja, estudar a deficiência é tão importante para as Ciências Humanas e Sociais quanto para os Estudos Feministas e de Gênero, especialmente quando se considera a deficiência um fenômeno/ experiência transversal a cada cultura, como o fazemos com gênero, raça/etnia, classe, entre outras categorias.

Do feminismo e dos Estudos Feministas e de Gênero, os Estudos sobre Deficiência trouxeram as contribuições de várias acadêmicas feministas estrangeiras. ${ }^{79}$ Há, inclusive, as que defendem estarem os Estudos sobre Deficiência percorrendo os mesmos caminhos que os Estudos Feministas e de Gênero, como se estivessem "inventando a roda" da crítica aos binarismos e reducionismos na reflexão em torno da relação do corpo com os preconceitos sociais e da imposição social de formas hegemônicas e hierárquicas de existência e subjetivação. Ao mesmo tempo, tais teóricas feministas da deficiência defendem a relevância da reflexão sobre a deficiência como categoria de análise fundamental ao avanço do campo acadêmico feminista. ${ }^{80}$

No Brasil os campos do feminismo e do gênero ainda estão engatinhando em relação ao recorte de deficiência 
${ }^{81}$ DINIZ, 2007, p. 59.

${ }^{82}$ ADRIÃO, 2008.

${ }^{83}$ Fúlvia ROSEMBERG, 1999; e Fúlvia ROSEMBERG e Leandro $\mathrm{F}$. ANDRADE, 2008.

${ }^{84}$ MORRIS, 2001.

${ }^{85}$ DINIZ e BARBOSA, 2010. em seus movimentos e estudos acadêmicos. Como enfatizou Diniz, ${ }^{81}$ em analogia ao sexismo, a luta dos movimentos da deficiência é contra a opressão do corpo deficiente. Essa preocupação fazia sentido, ainda que no Brasil não houvesse, num primeiro momento, debates sobre o tema da sexualidade das pessoas com deficiência, especialmente das mulheres com deficiência como novos sujeitos do feminismo brasileiro. Em todo caso, o pleno exercício da sexualidade das pessoas com deficiência dependia de resolver primeiro as questões da reabilitação do corpo lesado. Essa é uma das razões pelas quais as mulheres com deficiência em geral não têm participado expressivamente dos movimentos feministas no Brasil. ${ }^{82}$ Mulheres com deficiência são o tempo todo interpeladas a responderem primeiro às demandas desde seu lugar ou condição de deficiência, negligenciando ou deixando à margem seus outros sujeitos políticos. A outra possível razão de não se incluírem as questões da deficiência no feminismo deve-se ao inexpressivo número de mulheres com deficiência brasileiras nas universidades, uma vez que elas poderiam trazer do bojo dos movimentos da deficiência essa pauta para a academia.

A relação teórico-metodológica entre as dimensões de gênero e deficiência, contudo, deve respeitar o conceito de não sincronia ou heterocronia resgatado por Rosemberg. ${ }^{83}$ Nesse sentido, queremos afirmar a importância de se considerar a não linearidade da relação entre essas dimensões, uma vez que operam de modo muitas vezes hierárquico, não simultâneo entre si e entre as demais dimensões de idade, raça/etnia, classe, orientação sexual, dentre outras. Com base em Rosemberg, desejamos ressaltar que as diferentes formas de opressão não se efetivam da mesma forma e que o peso dessas formas de opressão nas histórias de vida e na análise de fenômenos sociais deve considerar suas distintas configurações sociais e históricas. Ademais, não pretendemos aqui sugerir a equivalência linear entre as formas de opressão de gênero e de deficiência. Como afirma Jenny Morris, ${ }^{84}$ a deficiência se distingue de outras formas de opressão pela "natureza aflitiva" dos corpos com lesões relacionadas, por exemplo, à artrite ou à epilepsia, que, diferentemente de outras condições como gênero, raça/etnia e classe, não são perturbadoras em si mesmas, mas operam como marcadores sociais da experiência de opressão. Não obstante, muitas pessoas com impedimentos corporais demandam recursos de reabilitação e intervenções sobre seus corpos numa lógica medicalizante, considerando indesejável a presença de algum impedimento sensorial, intelectual ou físico. ${ }^{85}$ Fatos como esses desafiam o modelo social da deficiência e 
${ }^{86}$ ADRIÃO, 2008.

${ }^{87}$ GARLAND-THOMSON, 2002.

${ }^{88} \mathrm{ASCH}, 2003$.

${ }^{89}$ BARROS, 2003.

${ }_{90}$ Agradecemos às revisoras anônimas pelas contribuições valiosas para qualificar o debate aqui proposto. podem ser mais bem pensados à luz das perspectivas feministas da deficiência, capazes de ampliar o potencial conceitual e abranger as múltiplas variações (inter)subjetivas produzidas na experiência de possuir um corpo com lesão.

As reflexões aqui desenvolvidas apontam para a importância da transversalidade da deficiência nas políicas de gênero, e vice-versa, o que nos convida a novas reflexões político-teóricas do feminismo, propondo-se a incorporação de demandas da área da deficiência, conforme já apontado por Adrião ${ }^{86}$ ao analisar também as questões singulares da deficiência na história do feminismo brasileiro. Com isso, espera-se contribuir para o enfrentamento às formas restritas de interpretação da variação corporal e de modos de vida, tema tão caro ao feminismo teórico e político e que ganha com a reflexão sobre a deficiência como categoria de análise um novo impulso conceitual. ${ }^{87}$

No entanto, nesse diálogo entre Estudos sobre Deficiência e Estudos Feministas e de Gênero, certamente, haverá pontos de tensão. Um deles se expressou no impacto que o texto de Asch, ${ }^{88}$ condenando o aborto seletivo a partir de pressupostos do modelo social da deficiência, teve entre acadêmicas feministas como Barros. ${ }^{89}$ Nesse caso, os pontos de tensão revelam diferenças em torno dos efeitos políticos que se pretendem forjar, em ambos os contextos, a partir dos argumentos e dos conceitos acadêmicos dos Estudos Feministas e de Gênero. ${ }^{90}$

\section{Referências}

ADRIÃO, Karla G. Encontros do feminismo: uma análise do campo feminista brasileiro a partir das esferas do movimento, do governo e da academia. 301 p. Tese (Doutorado Interdisciplinar em Ciências Humanas) - Universidade Federal de Santa Catarina, Florianópolis, 2008.

ALMASSI, Ben. "Disability, Functional Diversity, and Trans/ Feminism." International Journal of Feminist Approaches to Bioethics, v. 3, n. 2, p. 126-149, 2010.

AMARAL, Lígia A. Conhecendo a deficiência (em companhia de Hércules). São Paulo: Robe Editorial, 1995.

$\mathrm{ASCH}$, Adrienne. "Diagnóstico pré-natal e aborto seletivo: um desafio à prática e às políticas". Physis: Rev. Saúde Coletiva, v. 13, n. 2, p. 287-320, 2003.

"Critical Race Theory, Feminism, and Disability: Reflections on Social Justice and Personal Identity." In: SMITH, Bonnie G.; HUTCHISON, Beth (Org.). Gendering Disability. New Brunswick: Rutgers University Press, 2004. p. 9-44.

BARNES, Colin. "Disability Studies: New or not so New Directions?" Disability \& Society, v. 14, n. 4, p. 577-580, 1999. 
BARNES, Colin; OLIVER, Mike; BARTON, Len. Disability Studies Today. Cambridge: Polity Press, 2002.

BARROS, Alessandra. "Limites à condenação do aborto seletivo: a deficiência em contextos de países periféricos". Physis: Rev. Saúde Coletiva, v. 13, n. 2, p. 273-286, 2003.

BARTON, Len. "Sociologia y discapacidad: algunos temas nuevos". In: Discapacidad y sociedad. Madrid: Ediciones Morata; La Coruña: Fundación Paideia, 1998. p. 19-33.

BARTON, Len; OLIVER, Mike. "Introduction: The Birth of Disability Studies." In: . Disability Studies: Past, Present and Future. Leeds: The Disability Press, 1997. p. 12-16.

BEIGI, Amir B.; CHENG, Karen K. Y. "Rethinking Gender in Disability Issues." Sexuality and Disability, v. 28, n. 3, p. 205-207, 2010.

BRASIL. Decreto Legislativo n. 186, de 09 de julho de 2008. Aprova o texto da Convenção sobre os Direitos das Pessoas com Deficiência e de seu Protocolo Facultativo, assinados em Nova lorque, em 30 de março de 2007. Diário Oficial da União, Brasília-DF, 10 jul. 2008a, seção 1, edição 131, p. 1. Disponível em: <http://www2.senado. gov.br/bdst/item/id/99423 >. Acesso em: 21 jun. 2009.

. Convenção sobre os Direitos das Pessoas com Deficiência - Protocolo Facultativo à Convenção sobre os Direitos das Pessoas com Deficiência. Tradução Oficial/ Brasil. Brasília: Secretaria Especial de Direitos Humanos (SEDH); Coordenadoria Nacional para a Integração da Pessoa Portadora de Deficiência (Corde), 2008b.

. Política Nacional de Atenção Integral à Saúde do Homem. Brasília: Ministério da Saúde, 2010. Disponível em: <http://portal.saude.gov.br/portal/arquivos/pdf/ politica_nacional_homem.pdf > . Acesso em: 5 jun. 2010.

BROGNA, Pätricia. "El derecho a la igualdad... ¿O el derecho a la diferencia?". El Cotidiano, Universidad Autónoma Metropolitana - Azcapotzalco, Distrito Federal, México, v. 21, n. 134, p. 43-55, 2005.

BUTLER, Judith. Bodies That Matter: On the Discursive Limits of Sex. New York: Routledge, 1993.

Problemas de gênero: feminismo e subversão da identidade. Rio de Janeiro: Civilização Brasileira, 2003.

CALDERBANK, Rosemary. "Abuse and Disabled People: Vulnerability or Social Indifference?" Disability \& Society, v. 15, n. 3, p. 521-534, 2000.

CORKER, Mairian; SHAKESPEARE, Tom. (Eds.). Disability/Postmodernity: Embodying Disability Theory. London: Continuum, 2002.

DAVIS, Lennard. The Disability Studies Reader. New York: Routledge, 1997. 
DePAUW, Karen P. "'Space: The Final Frontier': The Invisibility of Disability on the Landscape of Women Studies." Frontiers: A Journal of Women Studies, v. 17, n. 3, p. 19-23, 1996.

DINIZ, Debora. "Modelo social da deficiência: a crítica feminista". Série Anis, v. 28, p. 1-8, jul. 2003a.

"Quem autoriza o aborto seletivo no Brasil? Médicos, promotores e juízes em cena". Physis: Rev. Saúde Coletiva, Rio de Janeiro, v. 13, n. 2, p. 13-34, 2003b.

. O que é deficiência? São Paulo: Brasiliense, 2007.

DINIZ, Debora; BARBOSA, Livia. "Pessoas com deficiência e direitos humanos no Brasil”. In: VENTURI, Gustavo (Org.). BRASIL. Presidência da República. Direitos Humanos: percepções da opinião pública - análises de pesquisa nacional. Brasília: Secretaria de Direitos Humanos, 2010. p. 207-217.

DINIZ, Debora; MEDEIROS, Marcelo; SQUINCA, Flávia "Reflexões sobre a versão em português da Classificação Internacional de Funcionalidade, Incapacidade e Saúde". Cad. Saúde Pública, Rio de Janeiro, v. 23, n. 10, p. 2507-2510, 2007

DINIZ, Debora; SQUINCA, Flávia; MEDEIROS, Marcelo. "Deficiência, cuidado e justiça distributiva". Série Anis, v. 48, p. 1-6, maio 2007.

DOUGLAS, Mary. Pureza e perigo. São Paulo: Perspectiva, 1976.

EREVELLES, Nirmala. "Disability and the Dialectics of Difference." Disability \& Society, v. 11, n. 4, p. 519-537, 1996.

FERRI, Beth A.; GREGG, Noel. "Women with Disabilities: Missing Voices." Women's Studies International Forum, v. 21 , n. 4 , p. 429-439, 1998.

FINE, Michelle; ASCH, Adrienne. Women with Disabilities: Essays in Psychology, Culture and Politics. Philadelphia: Temple University Press, 1988.

FINGER, Anne. "Forbidden Fruit." New Internationalist, n. 233, p. 8-10, 1992.

GARDOU, Charles. "Quais são os contributos da Antropologia para a compreensão das situações de deficiência?". Revista Lusófona de Educação, n. 8, p. 53-61, 2006.

GARLAND-THOMSON, Rosemarie. "Integrating Disability, Transforming Feminist Theory." NWSA Journal, v. 14, n. 3, p. 1-32, 2002.

Staring: How We Look. New York: Oxford University Press, 2009.

GERSCHICK, Thomas J. "Toward a Theory of Disability and Gender." Signs: Journal of Women in Culture \& Society, v. 25, n. 4, p. 1263-1268, 2000.

GLEESON, Brendan J. "Disability Studies: A Historical Materialist View." Disability \& Society, v. 12, n. 2, p. 179-202, 1997.

GOFFMAN, Erving. Estigma: notas sobre a manipulação da identidade deteriorada. Rio de Janeiro: LTC, 1988. 
GROSSI, Miriam P. "Feministas históricas e novas feministas no Brasil". Antropologia em Primeira Mão, n. 28, p. 1-34, 1998.

GUIMARÃES, Raquel. "Gênero e deficiência: um estudo sobre as relações de cuidado". In: DINIZ, Debora; SANTOS, Wederson (Org.). Deficiência e discriminação. Brasília: Letras Livres, 2010. p. 197-228.

HASSOUEH-PHILLIPS, Dena; CURRY, Mary A. "Abuse of Women with Disabilities: State of the Science." Rehabilitation Counseling Bulletin, v. 45, n. 2, p. 96-104, 2002.

HELMIUS, Gisela. "The Paradox of Discriminatory Practices as a Means of Emancipatory Strategies." Community, Work \& Family, v. 4, n. 3, p. 273-284, 2001.

HUGHES, Bill. "Disability and Body." In: BARNES, Colin; OLIVER, Mike; BARTON, Len (Org.). Disability Studies Today. Cambridge: Polity Press, 2002. p. 58-76.

"Wounded/Monstrous/Abject: A Critique of the Disabled Body in Sociological Imaginary." Disability \& Society, v. 24, n. 4, p. 399-410, 2009.

IBGE. Censo Demográfico 2010: resultados preliminares da amostra. Rio de Janeiro: IBGE, 2011. Disponível em: <http:/ /www.ibge.gov.br/home/estatistica/populacao/ censo2010/resultados_preliminares_amostra/ default_resultados_preliminares_amostra.shtm > . Acesso em: 21 nov. 2011.

KITTAY, Eva F. Love's Labor: Essays on Women, Equality and Dependency. New York: Routledge, 1999.

KITTAY, Eva F.; JENNINGS, Bruce; WASUNNA, Angela A. "Dependency, Difference and the Global Ethic of Longterm Care." The Journal of Political Philosophy, v. 13, n. 4, p. 443-469, 2005.

LLOYD, Margaret. "Does She Boil Eggs? Towards a Feminist Model of Disability." Disability, Handicap \& Society, v. 7, n. 3, p. 207-221, 1992.

MALUF, Sônia W. "Corpo e corporalidade nas culturas contemporâneas: abordagens antropológicas". Revista Esboços, v. 9, n. 9, p. 87-101, 2001.

MARTINS, Bruno Daniel G. S. "A angústia da transgressão corporal: a deficiência assim pensada". Oficina do CES, n. 237, p. 1-14, 2005.

MAYA, Asunción M. Mujer y discapacidad: una doble discriminación. Hergue: Andaluza; Huelva, 2004.

MAYS, Jennifer M. "Feminist Disability Theory: Domestic Violence against Women with a Disability." Disability \& Society, v. 21, n. 2, p. 147-158, 2006.

MELLO, Anahi G. "A construção da pessoa na experiência da deficiência: corpo, gênero, sexualidade, subjetividade e saúde mental". In: MALUF, Sônia W.; TORNQUIST, Carmen S. (Org.). Gênero, saúde e aflição: 
abordagens antropológicas. Florianópolis: Letras Contemporâneas, 2010. p. 133-191.

MORRIS, Jenny. Pride against Prejudice: Transforming Attitudes to Disability. London: The Women's Press, 1991. "Personal and Political: A Feminist Perspective in Researching Physical Disability." Disability, Handicap \& Society, v. 7, n. 2, p. 157-166, 1992.

. Encounters with Strangers: Feminism and Disability. London: The Women's Press, 1996.

"Impairment and Disability: Constructing an Ethics of Care that Promotes Human Rights." Hypatia, v. 16, n. 1, p. 1-16, 2001.

OLIVER, Mike. Social Work with Disabled People. Basingstoke: Macmillan, 1983.

."Social Policy and Disability: Some Theoretical Issues." Disability, Handicap \& Society, v. 1, n. 1, p. 5-17, 1986. 1990.

The Politics of Disablement. Basingstoke: Macmillan,

ORTEGA, Francisco. "Deficiência, autismo e neurodiversidade". Ciência \& Saúde Coletiva, v. 14 n. 1, p. 67-77, 2009.

PAIVA, Vera; PERES, Camila; BLESSA, Cely. "Jovens e adolescentes em tempos de aids: reflexões sobre uma década de trabalho de prevenção". Psicologia USP, v. 13, n. 1, p. 55-78, 2002.

PALACIOS, Agustina. El modelo social de discapacidad: orígenes, caracterización y plasmación en la Convención Internacional sobre los Derechos de las Personas con Discapacidad. Madrid: Cermi; Ediciones Cinca, 2008.

RANDOLPH, Diane; ANDRESEN, Elena Marie. "Disability, Gender, and Unemployment Relationships in the United States from the Behavioral Risk Factor Surveillance System." Disability \& Society, v. 19, n. 4, p. 403-414, 2004.

ROSEMBERG, Fúlvia. "Expansão da educação infantil e processos de exclusão". Cadernos de Pesquisa, n. 107, p. 7-40, 1999

ROSEMBERG, Fúlvia; ANDRADE, Leandro F. "Ação Afirmativa no ensino superior brasileiro: a tensão entre raça/etnia e gênero". Cadernos Pagu, n. 31, p. 420-437, 2008. Disponível em: <http://www.scielo.br/scielo.php?script= sci_arttext\&pid=S0104-8333200800020001 8\&Ing=en\& $\mathrm{nrm}=$ iso $>$. Acesso em: 27 jun. 2010.

SAMUELS, Ellen. "Critical Divides: Judith Butler's Body Theory and the Question of Disability." NWSA Journal, v. 14, n. 3, p. 58-76, 2002.

SASSAKI, Romeu K. Vida independente: história, movimento, liderança, conceito, filosofia e fundamentos; reabilitação, emprego e terminologia. São Paulo: RNR, 2003. 
SCHEER, Jessica. "Culture and Disability: An Anthropological Point of View." In: TRICKETT, Edison J.; WATTS, Roderick J.; BERMAN, Dina (Org.). Human Diversity: Perspectives on People in Context. San Francisco: Jossey-Bass, 1995. p. 244-260.

SCULLY, Jackie L. "Hidden Labor: Disabled/Nondisabled Encounters, Agency, and Autonomy." International Journal of Feminist Approaches to Bioethics, v. 3, n. 2, p. 25-42, 2010.

SHERRY, Mark. "Overlaps and Contradictions between Queer Theory and Disability Studies." Disability \& Society, v. 19, n. 7, p. 669-783, Dec. 2004.

SHAKESPEARE, Tom. "Poder y prejuicio: los temas de género sexualidad y discapacidad". In: BARTON, Len (Org.). Discapacidade y sociedad. Madrid: Moratta, 1998. p. 205-299.

"The Sexual Politics of Disabled Masculinity." Sexuality and Disability, v. 17, n. 1, p. 53-64, 1999.

SILVA, Otto M. A epopéia ignorada: a pessoa deficiente na história do mundo de ontem e hoje. São Paulo: Cedas, 1986.

SQUINCA, Flávia. "O debate sobre deficiência e cuidado como desafio para as teorias de justiça". Revista de Estudos Universitários, v. 34, n. 1, p. 57-69, 2008.

VASCONCELOS, Eduardo M. O poder que brota da dor e da opressão: empowerment, sua história, teorias e estratégias. São Paulo: Paulus, 2003.

VASH, Carolyn L. Enfrentando a deficiência: a manifestação, a psicologia, a reabilitação. São Paulo: Pioneira; EDUSP, 1988.

VERNON, Ayesha. "The Dialectics of Multiple Identities and the Disabled People's Movement." Disability \& Society, v. 14, n. 3, p. 385-398, 1999.

WENDELL, Susan. The Rejected Body: Feminist Philosophical Reflections on Disability. New York: Routledge, 1996.

WHO. World Report on Disability. Geneva: World Health Organization Press, 2011 . Disponível em: <http://whqlibdoc.who. int/publications/201 1/9789240685215_eng.pdf > . Acesso em: 22 nov. 2011.

WILLIAMS, Lúcia C. A. "Sobre deficiência e violência: reflexões para uma análise de revisão de área". Revista Brasileira de Educação Especial, v. 9, n. 2, p. 141-154, 2003.

[Recebido em 5 de julho de 2010, reapresentado em 22 de março de 2012 e aceito para publicação em 4 de junho de 2012] 


\section{Gender and Disability: Intersections and Perspectives}

Abstract: The field of feminist and gender studies has advanced while becoming linked with other categories of analysis, such as generation, class, race/ethnic groups, sexual orientation, region and religion. However, one issue still remains isolated from the effort of intersecting those categories, namely disability. Therefore, here we have sought to evaluate some of the possibilities to the analysis of social and cultural phenomena in which the dialog between feminist and gender studies and the field of disability studies is considered to be appropriate. It is expected that greater visibility will be provided to the debate on such issue by evaluating its analytical and political potential in view of its contribution to public policies.

Key Words: Gender; Disability; Public Policies. 